Editorial

\title{
Nanotechnology: Engineering Materials for a Better 21st Century
}

\author{
Meng Qiao, Xiyun Liu and Lei Zhang
}

Institute of Modern Optics, Nankai University, Tianjin 300071, P.R. China

Today, we are living in the midst of a technological revolution, where nanotechnology is demonstrating a greater commitment to make people's everyday lives better. The aim of nanotechnology is to create new materials with desired properties by engineering their nanoscale structures and components.

One of the most important impacts of nanotechnology is in medical diagnosis and treatment. Cardiovascular diseases such as heart disease and stroke are the leading causes of death and disability among men and women in almost every nation. There is a constant need for noninvasive, inexpensive and fast tools to diagnose these diseases. A recent progress is featured by the development of a test that used injected bionanomaterials to find diseased tissues and produce biomarkers in the urine. Detection of these biomarkers can be done within minutes using paper strips similar to a home pregnancy test. These bionanomaterials are composed of nanoparticles conjugated to cleavable ligand-encoded reporters. The reporters are engineered so that can be detected by sandwich immunoassays directly from the urine.

Among all diseases, cancer is still a major threat to human health this century. A valuable benefit of nanotechnology in cancer diagnostics is the development of nanosensors that can be used to detect cancer biomarkers with high sensitivity and low detection limit. In the case of prostate-specific antigen (PSA) detection for prostate cancer diagnosis, a novel plasmonic nanosensor based on the enzyme-guided coating of silver nanocrystals on gold nanostars is capable of detecting PSA down to $10^{-18} \mathrm{~g} \mathrm{~mL}^{-1}$ in whole serum. Label-free nanosensor technology for caner biomarker detection has also stimulated intense research.

Concerning nanotechnological applications in the treatment of cancer, the use of nanostructured materials in image-guided surgery for tumors is a forefront area. Though being the most efficient method to cure cancer, the completeness of tumor removal is still dependent on a surgeon's experience. To objectively delineate tumor margins during surgery, various kinds of nanomaterials have shown their usefulness as an imaging label to guide the surgery. A recent example accurately determined the brain tumor margins in living mice using a delicately designed triple-modality (magnetic resonance imagingphotoacoustic imaging-Raman imaging) nanoparticle. This, together with related works, gives researchers a decent idea of what to expect in human trials.

The application of nanotechnology in industry has also taken important steps. Enhanced Oil Recovery (EOR), also called tertiary oil recovery, aims to maximize oil recovery through extracting trapped oil from mature reservoirs. There is a strong thirst for new innovative materials to make EOR efforts more successful. It is noteworthy that a type of polymeric nanoparticle with the commercial name Bright Water has been field-tested in different oil reservoirs. These polymeric nanoparticles are thermal sensitive and could remarkably expand in volume within the reservoir, thus blocking the pore throats and redirecting the injected fluid to oil-rich zones.

Polymer-coated nanoparticles are an emerging class of materials that could offer a wealth of advantages for EOR due to improved solubility and stability, easier transport through porous media and greater stabilization of foams and emulsions. Other than coating with a polymer, surfactant-coated nanoparticles have also attracted considerable attention among researchers. The idea of using foam to reduce gas mobility during gas flooding has been proven effective in improving reservoir sweep efficiency. However, the inherent lack of long-term stability of foams limits the application of this technique. As a viable solution to this problem, modification of partially hydrophobic $\mathrm{SiO}_{2}$ nanoparticles with sodium dodecyl sulfate, a widely used surfactant, has taken a positive role in increasing the foam stability as a result of the adsorption of the particles on the bubble surface.

Molecular Deposition Film (MDF), sometimes described as a nanometer film, is another class of materials that are of current interest in EOR. MDF is capable of adhering to the rock surface due to electrostatic forces. The formation of this nanometer-thin film alters the properties of the rock surface from lipophilic to hydrophilic. This helps to separate the oil 
from the rock, enabling a more efficient displacement and recovery of the oil. It may be mentioned that MDF can hardly reduce the oil-water interfacial tension. Thus, a combined use of MDF and other flooding techniques would be a reasonable EOR practice.

As an ongoing research endeavor, nanosensors have been actively investigated as a tool to acquire oil reservoir information. Saudi Aramco, in collaboration with academic institutions, has developed nanomaterials that can sense real-time factors within the reservoir and transmit information such as the reservoir pressure, temperature and the fluid type to the operators when coming out with the oil from the well. Though the oil reservoir poses a challenging environment for this sensor technology, the development of these nanosensors shows a great prospect and is worthy of continued research attention.

Taken together, nanotechnology is a rapidly growing interdisciplinary technology with enormous potential for human health and industries. As a sustainable economic driver, nanotechnology can promise compelling social benefits. Despite of these advantages, the practical applications of this revolutionary technology are still a challenge.
For example, nanoparticles can agglomerate to form larger particles during the EOR process, compromising their function. Extensive and intensive research is apparently needed to overcome the shortcomings associated with the applications of nanotechnology. Nonetheless, the future of nanotechnology is bright. We would like to end this Editorial by quoting Walt Disney's "If you can dream it, you can do it." With the aid of nanotechnology, the dream of scientists to engineer new functional materials for a better $21 \mathrm{st}$ century is closer to a reality. 